\title{
Adjuvant systemic therapy in older women with breast cancer
}

This article was published in the following Dove Press journal:

Breast Cancer - Targets and Therapy

25 July 2016

Number of times this article has been viewed

Julieta Leone'

Bernardo Amadeo Leone'

José Pablo Leone ${ }^{2}$

'Department of Medical Oncology, Grupo Oncológico Cooperativo del Sur (GOCS), Neuquén, Argentina; ${ }^{2}$ Department of Internal Medicine, Division of Hematology-Oncology and Blood \& Marrow Transplantation, University of lowa Holden

Comprehensive Cancer Center, lowa City, IA, USA
Correspondence: José Pablo Leone University of lowa Hospitals and Clinics, 200 Hawkins Drive, C32 GH lowa City, IA 52242-108I, USA

$\mathrm{Tel}+\mathrm{I} 3193567839$

Fax +I 3193538383

Email jose-leone@uiowa.edu
Abstract: Breast cancer in the elderly is an increasing clinical problem. In addition, $\sim 60 \%$ of deaths from breast cancer occur in women aged 65 years and older. Despite this, older women with breast cancer have been underrepresented in clinical trials, and this has led to less than optimal evidence to guide their therapy. The management of elderly women with early breast cancer is a complex process that requires careful evaluation of life expectancy, comorbidities, patient values, and risks and benefits of available treatment options. This review will focus on current adjuvant systemic therapy options for older women with breast cancer, discuss the principles in the decision-making process, and define the role of endocrine therapy, chemotherapy, and targeted agents.

Keywords: breast cancer, adjuvant therapy, chemotherapy, endocrine therapy, elderly

\section{Introduction}

Breast cancer is a disease that increases in incidence with aging. In the US, the average age of diagnosis is at 61 years of age and $60 \%$ of breast cancer deaths occur in women aged 65 and older. ${ }^{1}$ The US population is aging, and in 2030, the proportion of adults 65 years and older is projected to rise from $14 \%$ to $20 \%$ of the total US population. ${ }^{2}$ Due to the aging of the population and the consistent observation that cancer incidence rises dramatically with age, it is anticipated that between 2010 and 2030, there will be a $67 \%$ increase in cancer incidence for the population aged 65 years and older. ${ }^{3}$ Despite the increased incidence of breast cancer among older women, unfortunately, there is insufficient clinical trial data to guide treatment decisions. It is of particular concern that the available evidence suggests that older patients are less likely to receive standard therapies. ${ }^{4,5}$ Furthermore, elderly patients may be more likely to discontinue these treatments. ${ }^{6}$ Underutilization of standard therapies coupled with their early discontinuation are likely the main reasons behind the lesser mortality benefits seen in older patients compared with their younger counterparts, ${ }^{7}$ despite the generally favorable tumor biology in the older population. ${ }^{8}$

The majority of older patients are diagnosed with early-stage breast cancer. This represents a unique opportunity where systemic therapies are considered in order to increase the chances of cure. ${ }^{9}$ Because elderly patients often have other comorbidities, treatment decisions represent a difficult challenge that requires careful consideration of age heterogeneity, expected benefit from therapy, and estimated life expectancy. This review will focus on the key issues of current adjuvant systemic therapy options for older women with breast cancer, comment on the principles in the decision process, and define the role of specific options including endocrine therapy, chemotherapy, and targeted agents. 


\section{Endocrine therapy}

Most elderly patients with breast cancer will present with estrogen receptor-positive, progesterone receptor-positive, and human epidermal growth factor receptor 2 (HER2)-negative tumors at diagnosis. These patients are the best candidates for endocrine therapy as the mainstay adjuvant systemic therapy. This also applies to the subgroup of patients with substantial comorbidities and life expectancy of only several years. The efficacy of endocrine therapy has no correlation with age. ${ }^{10}$ There are two main options for endocrine therapy: tamoxifen and aromatase inhibitors. In the BIG 1-98 (Breast International Group) trial, the aromatase inhibitor letrozole showed age-independent superior efficacy compared with tamoxifen, with improvements in local and distant control and overall survival (OS). ${ }^{11}$ The ATAC (Arimidex, Tamoxifen, Alone or in Combination) trial showed similar findings in favor of anastrozole compared with tamoxifen, with fewer thrombotic and uterine cancer episodes, even after a median follow-up of 100 months. ${ }^{12}$ Finally, the MA.17 trial showed that disease-free survival (DFS) and OS improved in all age groups with the use of letrozole after 5 years of tamoxifen compared with placebo after 5 years of tamoxifen. ${ }^{13}$ Therefore, aromatase inhibitors are favored over tamoxifen due to their superior outcomes and more favorable adverse event profile in the elderly population, ${ }^{14}$ in particular, by avoiding the feared risks of venous thromboembolism and uterine cancer that are associated with tamoxifen use. ${ }^{15}$ Current data supports the use of aromatase inhibitors for at least 5 years. ${ }^{16}$ Ongoing trials are evaluating the benefit of continuing therapy beyond 5 years.

Side effects of aromatase inhibitors include arthralgias and myalgias that can affect patient functioning and lead to discontinuation of therapy. ${ }^{17}$ In the exploratory analysis of ATAC, $35.2 \%$ of women treated with anastrozole developed joint symptoms. ${ }^{18}$ Development of osteoporosis and bone fractures has also been associated with the use of aromatase inhibitors. ${ }^{19,20}$ Elderly patients treated with aromatase inhibitors should be instructed to take daily calcium supplementation with vitamin $\mathrm{D}$ and advised to do regular exercise and physical activity. If patients develop osteoporosis while on therapy with aromatase inhibitors, treatment with bisphosphonates or denosumab should be initiated. ${ }^{21}$ In contrast, tamoxifen has a protective effect against bone loss and fractures, which has been well documented in the older population. ${ }^{22}$

The role of bisphosphonates and denosumab as adjuvant systemic therapy in early breast cancer has gained attention in recent years due to potential antitumor activity and improvements in bone health. A recent meta-analysis evaluated the use of adjuvant bisphosphonates and reported that among
11,767 postmenopausal women, the treatment resulted in significant reduction in bone recurrence and breast cancer mortality. ${ }^{23}$ The ABCSG-18 trial randomized 3,425 postmenopausal, aromatase inhibitor-treated women with early breast cancer to denosumab or placebo. The study showed a significant reduction in the risk of clinical fractures in the denosumab group with acceptable toxicity. ${ }^{24}$ These data support the use of bisphosphonates or denosumab in the adjuvant therapy of elderly women who are receiving aromatase inhibitors; however, certain factors should be considered prior to initiating this treatment, such as the risk of severe complications - osteonecrosis of the jaw and renal failure, cost of therapy, and life expectancy.

In patients with a short life expectancy in whom surgery may not be feasible, primary endocrine therapy can effectively control tumor growth for around 18-24 months..$^{25}$ Although time to onset of a response may take several months, ${ }^{26}$ many women will benefit from continuous disease control throughout their life span. It is important to notice, though, that surgery is a very safe intervention in elderly women ${ }^{27}$ and remains the standard of care in breast cancer for patients of any age, except in those who are not surgical candidates, have limited life expectancy, or do not want surgery. ${ }^{28}$

In patients with very small tumors ( $\leq 5 \mathrm{~mm}$ ) or those with multiple comorbidities, endocrine treatment could be spared. ${ }^{9}$ Data from a population-based cohort study suggest that omitting adjuvant systemic therapy showed similar OS as compared to the general population for women aged 60-74 years with $\leq 10 \mathrm{~mm}$, node-negative, hormone-sensitive, and grade 1 ductal carcinoma or grade 1 or 2 lobular carcinoma. ${ }^{29}$

In addition to monitoring toxicities during treatment, it is crucial to evaluate compliance to therapy as nonadherence to adjuvant endocrine therapy is associated with increased mortality in breast cancer patients. ${ }^{6}$ Counseling patients about the importance of treatment compliance should be done on a regular basis as nonadherence has been reported to occur in a high proportion of cases, with around half of the patients not finishing 5 years of treatment. ${ }^{30,31}$ Elderly patients are more likely to decline the recommended endocrine therapy, especially if they are treated by a nononcologist physician. ${ }^{32}$

\section{Chemotherapy}

The first step in the evaluation of older women with breast cancer who are being evaluated for systemic chemotherapy is to estimate their life expectancy. This is a complex but important assessment that considers the patient's age, performance status, and comorbidities. Online models such as ePrognosis that consider medical as well as functional variables can be 
a useful resource to estimate life expectancy. Older patients are a highly heterogeneous group with regards to their health and functional status, and because of this, assessments of life expectancy based on chronological age alone are notoriously unreliable for making treatment decisions. In general, regardless of stage, patients with luminal A breast cancers with poor life expectancy should not be recommended chemotherapy. In contrast, for patients with $>5$ years of life expectancy or patients with triple-negative or HER2-positive breast cancer, chemotherapy may be an important component of their care.

After a careful evaluation of life expectancy, the main question is how much benefit would be obtained with the administration of chemotherapy. Online tools (Adjuvant! and PREDICT) can assist with the estimation of benefit of administration of systemic therapy. ${ }^{33,34}$ Both models have been validated and are similarly accurate for predicting treatment benefit. The PREDICT model can reliably assess the benefit of treatment in HER2-positive patients, whereas Adjuvant! takes comorbidities into consideration and is particularly helpful in patients with multiple comorbidities. Both models can be used to guide decisions on adjuvant chemotherapy; in most patients, chemotherapy should not be indicated if the predicted benefit in OS at 10 years is $<3 \%$, should be considered if improvement in OS is between $3 \%$ and $5 \%$, and should be recommended if the benefit is $>5 \%$. In addition to these two models, multigene prognostic assays such as oncotype Dx and mammaprint, when used in the appropriate setting and patient population, can provide estimates on the predictive benefit of chemotherapy based on specific molecular and genetic features of a patient's tumor. ${ }^{35,36}$ Nonetheless, when using these tools in older patients, careful consideration should be given to individual patient's life expectancy and tumor biomarkers, as predicting risk of breast cancer recurrence or death at 10 years may not be helpful in two groups of patients: 1) those who have too many comorbidities who could die in a much shorter time from other diseases, and 2) those who are receiving adjuvant endocrine therapy who may experience breast cancer relapse at a much later time. ${ }^{37}$

There are many options for chemotherapy regimens that can be used in older women with breast cancer. These include docetaxel plus cyclophosphamide, ${ }^{38}$ fluorouracil plus epirubicin and cyclophosphamide, ${ }^{39}$ adriamycin with cyclophosphamide (AC) followed by paclitaxel, ${ }^{40}$ among others. Potential treatment-related toxicities are very important when choosing a particular chemotherapy regimen. Cyclophosphamide plus methotrexate and fluorouracil (CMF) can be considered in selected patients, particularly those with decreased cardiac function, and has shown to be superior to capecitabine in a large randomized clinical trial of elderly patients with earlystage breast cancer. ${ }^{41}$ However, only $60 \%$ of patients were able to complete six cycles of oral-based CMF, whereas AC was generally well tolerated. ${ }^{41} \mathrm{~A}$ potential strategy to decrease toxicity and obtain better compliance could be the administration of modified $\mathrm{CMF}^{42}$ Anthracycline-containing regimens have shown similar benefits independent of age. ${ }^{43}$ However, rates of cardiac toxicity and therapy-related myelodysplasia or acute leukemia are higher in patients $>65$ years old. ${ }^{44} 46$ For elderly patients in whom even mild neuropathy may lead to functional impairment, the use of regimens that do not contain taxanes might be preferred.

There are several clinical trials that evaluated the role of chemotherapy in older women with breast cancer. The French Adjuvant Study Group 08 trial evaluated fit women $>65$ years with node-positive early breast cancer. Patients were randomized to tamoxifen with or without weekly epirubicin; the multivariate analysis showed a significantly higher relative risk of relapse of 1.93 (95\% confidence interval [CI]: 1.70-2.17) with tamoxifen compared with the combination $(P=0.005){ }^{47}$

The Cancer and Leukemia Group B 49907 trial was a noninferiority study that compared adjuvant $\mathrm{AC}$ or $\mathrm{CMF}$ against single-agent capecitabine in women aged 65 years or older with stage I to IIIB breast cancer. The hazard ratio for disease recurrence or death in the capecitabine group was 2.09 (95\% CI: $1.38-3.17 ; P<0.001)$. In addition, the combination chemotherapy showed significantly improved DFS and OS. ${ }^{41} \mathrm{~A}$ subsequent report on quality of life showed that capecitabine monotherapy had significantly better quality of life, role function, social function, fewer systemic adverse effects, less psychological distress, and less fatigue, compared to combination chemotherapy with $\mathrm{AC}$ or $\mathrm{CMF} .{ }^{48}$ However, all these differences resolved by 12 months, arguing that the short-term toxicity from the standard chemotherapy may be an acceptable trade-off for an enhanced survival advantage. ${ }^{48}$

In the elderly Docetaxel Adjuvant trial, elderly women aged 65 years or older with node-positive or high-risk node-negative tumors were randomized to CMF or weekly docetaxel. The study showed no significant differences in DFS or OS for either group; however, docetaxel produced worse quality of life and adverse effects, in general. ${ }^{49}$ Finally, a recent study randomized 400 women aged 65 years or older with high-risk early breast cancer to standard chemotherapy with either EC or CMF versus the experimental arm which consisted of six cycles of nab-paclitaxel and capecitabine. The study showed no differences in survival between the treatment groups; however, the experimental arm led to more treatment discontinuations and nonhematological toxicities. ${ }^{50}$ 
Although adjuvant systemic chemotherapy has clearly improved survival in older women, these patients should be closely monitored during therapy as they are more susceptible to toxicities. Because older patients have higher risk of developing neutropenia, primary prophylaxis with granulocyte colony-stimulating factor should be discussed and may be indicated before initiating chemotherapy, ${ }^{51}$ especially in rural areas. Adjuvant chemotherapy in elderly patients can be associated with a risk of hospitalization. A population-based analysis has shown that frequently used chemotherapy regimens can lead to considerable rates of hospitalizations, particularly in patients $>65$ years. ${ }^{52}$ However, only $<1 \%$ of patients died due to complications from chemotherapy. The risk of hospitalization is an important factor to consider when recommending adjuvant chemotherapy, as hospitalization in elderly women is associated with significant decline in function and quality of life. ${ }^{53}$ Certain tools, such as the Chemotherapy Risk Assessment Scale for High-Age Patient score, are helpful to predict the risk of toxicity from chemotherapy and assist in treatment selection. $^{54}$

Finally, it is very appropriate to discuss treatment goals and expectations of elderly patients when deciding between specific treatments. A study evaluating patients $>60$ years reported that $74.4 \%$ and $88.8 \%$ would prefer to die than experience severe treatment-related functional impairment or cognitive impairment, respectively ${ }^{55}$ In addition, the study reported that the number of participants who would choose treatment declined as the likelihood of an adverse outcome increased. ${ }^{55}$ While young patients considering systemic therapy will primarily focus on advantages in DFS and OS, older patients give priority to remain functionally active and independent for as long as possible.

\section{Trastuzumab}

Previous studies suggested that HER2-positive breast cancer appears to be uncommon in older patients in comparison with their younger counterparts. ${ }^{56,57}$ However, this finding has not been validated. ${ }^{58}$ Current data supports that, regardless of age, survival outcomes are worse in HER2positive compared with HER2-negative tumors. ${ }^{59}$ Despite this, recommending chemotherapy plus trastuzumab in elderly patients can be a difficult decision. These patients frequently have several comorbidities that increase the risk of toxicity and death..$^{60}$ Therefore, prior to the recommendation of chemotherapy with trastuzumab, a careful evaluation is strongly advised, particularly when one considers that cardiac toxicity is one of the most important adverse events from trastuzumab. ${ }^{61}$
Several large randomized clinical trials have confirmed the efficacy of trastuzumab in the adjuvant therapy of HER2positive breast cancer. ${ }^{62-64}$ However, in these studies, older women have been inadequately represented. In the NSABPB31, NCCTC-N9831, and HERA trials, only $16 \%$ of patients were 60 years or older. ${ }^{62,63}$ In addition, in other European trials, women over 65 years were excluded. ${ }^{65,66}$

In the joint analysis of NCCTC-N9831 and NSABPB31 trials, women over 60 years for whom trastuzumab was added to their therapy, experienced improvement in OS with a hazard ratio of $0.41 .^{62}$ This benefit was similar to the one seen in the overall cohort. In contrast, the HERA trial reported no differences in elderly women between the trastuzumab and the observation groups (hazard ratio: 0.91 ; 95\% CI: 0.59-1.41); however, this study was underpowered for this comparison and the sequential administration of trastuzumab as opposed to concurrent administration may have resulted in less efficacy. ${ }^{63,67,68}$ Finally, the Breast Cancer International Research Group 006 study showed that the addition of trastuzumab to two different chemotherapy regimens led to a significant improvement in OS. ${ }^{64}$ In this trial, the docetaxel plus carboplatin plus trastuzumab regimen showed similar efficacy for DFS and OS, compared with the standard anthracycline-trastuzumab regimen, but with lesser cardiac toxicity ${ }^{64}$ However, the study excluded patients $>70$ years, thereby limiting conclusions in this population.

Recently, a single-arm, Phase II study evaluated the combination of weekly paclitaxel with trastuzumab in 406 patients with small $(<3 \mathrm{~cm})$, node-negative, HER2-positive tumors. ${ }^{69}$ In this trial, $34 \%$ of patients were aged 60 years or older. Despite the lack of a control arm, the study reported an impressive 3-year DFS rate of $98.7 \%$ with $0.5 \%$ incidence of symptomatic heart failure, making this combination particularly attractive to elderly patients.

With regards to cardiac safety, both NSABP-B31 and NCCTC-N9831 have shown higher risk of cardiac toxicity in the elderly. ${ }^{70,71}$ In particular, NSABP-B31 reported that the risk of congestive heart failure was 2.5 times higher in patients $>60$ years compared to their younger counterparts. ${ }^{70}$ In line with these results, NCCTC-N9831 described that the risk for congestive heart failure was 3.5 times higher for this subgroup of patients. ${ }^{71}$ The joint analysis of these two trials with longer follow-up confirmed these initial findings and showed a hazard ratio of $3.0(P=0.0022)$ in patients over 50 years compared with those $<50 .{ }^{72}$ In contrast, HERA showed no differences in cardiac toxicity between age groups. ${ }^{73}$

Based on the available data, incorporation of trastuzumab should be recommended in the adjuvant treatment of elderly 
women with HER2-positive breast cancer, particularly if patients have normal cardiac function, no history of cardiac diseases, and the treatment is deemed beneficial. One year of trastuzumab, preferably given in combination rather than in sequence with chemotherapy, represents the standard of care. ${ }^{68,74}$ A preferred alternative for certain patients could be the combination of trastuzumab with nonanthracycline chemotherapy, which is associated with less cardiac toxicity. ${ }^{64}$ Another potential option, especially for patients with node-negative tumors who may not be good candidates for polychemotherapy, is the administration of weekly paclitaxel with trastuzumab, which is an effective and well-tolerated treatment. ${ }^{69}$ After trastuzumab initiation, it is important to closely monitor for early symptoms and cardiac function, as most cardiac events related to trastuzumab are reversible. ${ }^{75}$

Currently, there is insufficient literature available to comment on the use of adjuvant trastuzumab in combination with endocrine therapy or as monotherapy, which could be an important therapeutic strategy for selected older patients with HER2-positive disease due to its lower toxicity. RESPECT (N-SAS BC07), a Phase 3 randomized study evaluating the efficacy and safety of trastuzumab monotherapy compared with standard trastuzumab in combination with chemotherapy, is ongoing. ${ }^{76}$ The study is enrolling women over 70 years with HER2-positive breast cancer and the results are eagerly awaited as they will be particularly helpful in the management of elderly patients.

\section{Conclusion}

The management of elderly women with breast cancer is highly complex. Unfortunately, limited data are available from clinical trials to guide practice, and therefore, randomized controlled trials in older women are urgently needed since breast cancer in older women will become an increasing problem in the years to come. Patients should be actively involved in the decision-making process of their therapy, as elderly patients may often favor goals of quality of life and independence. Nonetheless, standard adjuvant treatments should be offered to fit elderly patients irrespective of age, and physicians should inform their patients that undertreatment strongly decreases the prognosis of breast cancer. Therefore, the decisions about adjuvant therapy should be made in conjunction with the patient, to respect their preferences and discuss available options.

\section{Acknowledgment}

We want to thank the University of Iowa Libraries for the support provided for this publication.

\section{Disclosure}

The authors report no conflicts of interest in this work.

\section{References}

1. SEER Cancer Statistics Factsheets: Breast Cancer. Bethesda, MD: National Cancer Institute; 2015.

2. Ortman JM, Velkoff VA, Howard H. An aging nation: the older population in the United States. Washington, DC: US Census Bureau; 2014.

3. Smith BD, Smith GL, Hurria A, Hortobagyi GN, Buchholz TA. Future of cancer incidence in the United States: burdens upon an aging, changing nation. J Clin Oncol. 2009;27(17):2758-2765.

4. Lavelle K, Todd C, Moran A, Howell A, Bundred N, Campbell M. Non-standard management of breast cancer increases with age in the UK: a population based cohort of women $>$ or $=65$ years. Br J Cancer 2007;96(8):1197-1203.

5. Giordano SH, Hortobagyi GN, Kau SW, Theriault RL, Bondy ML. Breast cancer treatment guidelines in older women. J Clin Oncol. 2005;23(4):783-791.

6. Hershman DL, Shao T, Kushi LH, et al. Early discontinuation and nonadherence to adjuvant hormonal therapy are associated with increased mortality in women with breast cancer. Breast Cancer Res Treat. 2011;126(2):529-537.

7. Bouchardy C, Rapiti E, Fioretta G, et al. Undertreatment strongly decreases prognosis of breast cancer in elderly women. J Clin Oncol. 2003;21(19):3580-3587.

8. Diab SG, Elledge RM, Clark GM. Tumor characteristics and clinical outcome of elderly women with breast cancer. $J$ Natl Cancer Inst. 2000;92(7):550-556.

9. Biganzoli L, Wildiers H, Oakman C, et al. Management of elderly patients with breast cancer: updated recommendations of the International Society of Geriatric Oncology (SIOG) and European Society of Breast Cancer Specialists (EUSOMA). Lancet Oncol. 2012;13(4):e148-e160.

10. Owusu C, Lash TL, Silliman RA. Effectiveness of adjuvant tamoxifen therapy among older women with early stage breast cancer. Breast $J$. 2007;13(4):374-382.

11. Colleoni M, Giobbie-Hurder A, Regan MM, et al. Analyses adjusting for selective crossover show improved overall survival with adjuvant letrozole compared with tamoxifen in the BIG 1-98 study. J Clin Oncol. 2011;29(9):1117-1124.

12. Forbes JF, Cuzick J, Buzdar A, Howell A, Tobias JS, Baum M. Effect of anastrozole and tamoxifen as adjuvant treatment for early-stage breast cancer: 100-month analysis of the ATAC trial. Lancet Oncol. 2008;9(1):45-53.

13. Goss PE, Ingle JN, Martino S, et al. Randomized trial of letrozole following tamoxifen as extended adjuvant therapy in receptor-positive breast cancer: updated findings from NCIC CTG MA.17. J Natl Cancer Inst. 2005;97(17):1262-1271.

14. Muss HB, Tu D, Ingle JN, et al. Efficacy, toxicity, and quality of life in older women with early-stage breast cancer treated with letrozole or placebo after 5 years of tamoxifen: NCIC CTG intergroup trial MA.17. J Clin Oncol. 2008;26(12):1956-1964.

15. Amir E, Seruga B, Niraula S, Carlsson L, Ocana A. Toxicity of adjuvant endocrine therapy in postmenopausal breast cancer patients: a systematic review and meta-analysis. J Nati Cancer Inst. 2011;103(17):1299-1309.

16. Senkus E, Kyriakides S, Penault-Llorca F, et al. Primary breast cancer: ESMO Clinical Practice Guidelines for diagnosis, treatment and followup. Ann Oncol. 2013;24 Suppl 6:vi7vi23.

17. Ziller V, Kalder M, Albert US, et al. Adherence to adjuvant endocrine therapy in postmenopausal women with breast cancer. Ann Oncol. 2009;20(3):431-436.

18. Sestak I, Cuzick J, Sapunar F, et al. Risk factors for joint symptoms in patients enrolled in the ATAC trial: a retrospective, exploratory analysis. Lancet Oncol. 2008;9(9):866-872.

19. Baum M, Budzar AU, Cuzick J, et al. Anastrozole alone or in combination with tamoxifen versus tamoxifen alone for adjuvant treatment of postmenopausal women with early breast cancer: first results of the ATAC randomised trial. Lancet. 2002;359(9324):2131-2139. 
20. Neuner JM, Yen TW, Sparapani RA, Laud PW, Nattinger AB. Fracture risk and adjuvant hormonal therapy among a population-based cohort of older female breast cancer patients. Osteoporosis Int. 2011;22(11):2847-2855.

21. Eidtmann H, de Boer R, Bundred N, et al. Efficacy of zoledronic acid in postmenopausal women with early breast cancer receiving adjuvant letrozole: 36-month results of the ZO-FAST Study. Ann Oncol. 2010;21(11):2188-2194.

22. Koopal C, Janssen-Heijnen ML, van de Wouw AJ, van den Bergh JP. Fracture incidence in pre- and postmenopausal women after completion of adjuvant hormonal therapy for breast cancer. Breast. 2015;24(2):153-158.

23. Coleman R, Powles T, Paterson A, et al. Adjuvant bisphosphonate treatment in early breast cancer: meta-analyses of individual patient data from randomised trials. Lancet. 2015;386(10001):1353-1361.

24. Gnant M, Pfeiler G, Dubsky PC, et al. Adjuvant denosumab in breast cancer (ABCSG-18): a multicentre, randomised, double-blind, placebocontrolled trial. Lancet. 2015;386(9992):433-443.

25. Hind D, Wyld L, Beverley CB, Reed MW. Surgery versus primary endocrine therapy for operable primary breast cancer in elderly women (70 years plus). Cochrane Database Syst Rev. 2006;25(1):CD004272.

26. Dixon JM, Renshaw L, Macaskill EJ, et al. Increase in response rate by prolonged treatment with neoadjuvant letrozole. Breast Cancer Res Treat. 2009;113(1):145-151.

27. Chatzidaki P, Mellos C, Briese V, Mylonas I. Perioperative complications of breast cancer surgery in elderly women ( $>/=80$ years). Ann Surg Oncol. 2011;18(4):923-931.

28. Hind D, Wyld L, Reed MW. Surgery, with or without tamoxifen, vs tamoxifen alone for older women with operable breast cancer: Cochrane review. Br J Cancer. 2007;96(7):1025-1029.

29. Christiansen P, Bjerre K, Ejlertsen B, et al. Mortality rates among earlystage hormone receptor-positive breast cancer patients: a populationbased cohort study in Denmark. J Natl Cancer Inst. 2011;103(18): 1363-1372.

30. Schlenk EA, Dunbar-Jacob J, Engberg S. Medication non-adherence among older adults: a review of strategies and interventions for improvement. J Gerontol Nurs. 2004;30(7):33-43.

31. Hershman DL, Kushi LH, Shao T, et al. Early discontinuation and nonadherence to adjuvant hormonal therapy in a cohort of 8,769 early-stage breast cancer patients. J Clin Oncol. 2010;28(27):4120-4128.

32. Guth U, Myrick ME, Kandler C, Vetter M. The use of adjuvant endocrine breast cancer therapy in the oldest old. Breast. 2013;22(5):863-868.

33. Ravdin PM, Siminoff LA, Davis GJ, et al. Computer program to assist in making decisions about adjuvant therapy for women with early breast cancer. J Clin Oncol. 2001;19(4):980-991.

34. Wishart GC, Bajdik CD, Dicks E, et al. PREDICT Plus: development and validation of a prognostic model for early breast cancer that includes HER2. Br J Cancer. 2012;107(5):800-807.

35. Paik S, Shak S, Tang G, et al. A multigene assay to predict recurrence of tamoxifen-treated, node-negative breast cancer. $N$ Engl J Med. 2004; 351(27):2817-2826.

36. Fan C, Oh DS, Wessels L, et al. Concordance among gene-expressionbased predictors for breast cancer. N Engl J Med. 2006;355(6):560-569.

37. Effects of chemotherapy and hormonal therapy for early breast cancer on recurrence and 15-year survival: an overview of the randomised trials. Lancet. 2005;365(9472):1687-1717.

38. Jones S, Holmes FA, O'Shaughnessy J, et al. Docetaxel with cyclophosphamide is associated with an overall survival benefit compared with doxorubicin and cyclophosphamide: 7-year follow-up of US Oncology Research Trial 9735. J Clin Oncol. 2009;27(8):1177-1183.

39. Martin M, Rodriguez-Lescure A, Ruiz A, et al. Randomized phase 3 trial of fluorouracil, epirubicin, and cyclophosphamide alone or followed by Paclitaxel for early breast cancer. J Natl Cancer Inst. 2008; 100(11):805-814.

40. Sparano JA, Wang M, Martino S, et al. Weekly paclitaxel in the adjuvant treatment of breast cancer. $N$ Engl J Med. 2008;358(16):1663-1671.

41. Muss HB, Berry DA, Cirrincione CT, et al. Adjuvant chemotherapy in older women with early-stage breast cancer. $N$ Engl J Med. 2009;360(20):2055-2065
42. Poole CJ, Earl HM, Hiller L, et al. Epirubicin and cyclophosphamide, methotrexate, and fluorouracil as adjuvant therapy for early breast cancer. N Engl J Med. 2006;355(18):1851-1862.

43. Muss HB, Woolf S, Berry D, et al. Adjuvant chemotherapy in older and younger women with lymph node-positive breast cancer. JAMA. 2005;293(9):1073-1081.

44. Doyle JJ, Neugut AI, Jacobson JS, Grann VR, Hershman DL. Chemotherapy and cardiotoxicity in older breast cancer patients: a populationbased study. J Clin Oncol. 2005;23(34):8597-8605.

45. Wolff AC, Blackford AL, Visvanathan K, et al. Risk of marrow neoplasms after adjuvant breast cancer therapy: the national comprehensive cancer network experience. J Clin Oncol. 2015;33(4):340-348.

46. Howard RA, Gilbert ES, Chen BE, et al. Leukemia following breast cancer: an international population-based study of 376,825 women. Breast Cancer Res Treat. 2007;105(3):359-368.

47. Fargeot P, Bonneterre J, Roche H, et al. Disease-free survival advantage of weekly epirubicin plus tamoxifen versus tamoxifen alone as adjuvant treatment of operable, node-positive, elderly breast cancer patients: 6-year follow-up results of the French adjuvant study group 08 trial. $J$ Clin Oncol. 2004;22(23):4622-4630.

48. Kornblith AB, Lan L, Archer L, et al. Quality of life of older patients with early-stage breast cancer receiving adjuvant chemotherapy: a companion study to cancer and leukemia group B 49907. J Clin Oncol. 2011;29(8):1022-1028.

49. Perrone F, Nuzzo F, Di Rella F, et al. Weekly docetaxel versus CMF as adjuvant chemotherapy for older women with early breast cancer: final results of the randomized phase III ELDA trial. Ann Oncol. 2015;26(4):675-682.

50. von Minckwitz G, Conrad B, Reimer T, et al. A randomized phase 2 study comparing EC or CMF versus nab-paclitaxel plus capecitabine as adjuvant chemotherapy for nonfrail elderly patients with moderate to high-risk early breast cancer (ICE II-GBG 52). Cancer. 2015;121(20):3639-3648.

51. Balducci L, Al-Halawani H, Charu V, et al. Elderly cancer patients receiving chemotherapy benefit from first-cycle pegfilgrastim. Oncologist. 2007;12(12):1416-1424.

52. Barcenas $\mathrm{CH}$, Niu J, Zhang N, et al. Risk of hospitalization according to chemotherapy regimen in early-stage breast cancer. J Clin Oncol. 2014;32(19):2010-2017.

53. Hoogerduijn JG, Buurman BM, Korevaar JC, Grobbee DE, de Rooij SE, Schuurmans MJ. The prediction of functional decline in older hospitalised patients. Age Ageing. 2012;41(3):381-387.

54. Extermann M, Boler I, Reich RR, et al. Predicting the risk of chemotherapy toxicity in older patients: the Chemotherapy Risk Assessment Scale for High-Age Patients (CRASH) score. Cancer. 2012;118(13): 3377-3386.

55. Fried TR, Bradley EH, Towle VR, Allore H. Understanding the treatment preferences of seriously ill patients. NEngl J Med. 2002;346(14): 1061-1066.

56. Molino A, Giovannini M, Auriemma A, et al. Pathological, biological and clinical characteristics, and surgical management, of elderly women with breast cancer. Crit Rev Oncol Hematol. 2006;59(3):226-233.

57. Daidone MG, Coradini D, Martelli G, Veneroni S. Primary breast cancer in elderly women: biological profile and relation with clinical outcome. Crit Rev Oncol Hematol. 2003;45(3):313-325.

58. Mustacchi G, Cazzaniga ME, Pronzato P, De Matteis A, Di Costanzo F, Floriani I. Breast cancer in elderly women: a different reality? Results from the NORA study. Ann Oncol. 2007;18(6):991-996.

59. Engels CC, Kiderlen M, Bastiaannet E, et al. The clinical value of HER-2 overexpression and PIK3CA mutations in the older breast cancer population: a FOCUS study analysis. Breast Cancer Res Treat. 2016;156(2):361-370.

60. Muss HB. Coming of age: breast cancer in seniors. Oncologist. 2010;15(Suppl 5):57-65.

61. Boekhout AH, Beijnen JH, Schellens JH. Trastuzumab. Oncologist. 2011;16(6):800-810.

62. Romond EH, Perez EA, Bryant J, et al. Trastuzumab plus adjuvant chemotherapy for operable HER2-positive breast cancer. N Engl J Med. 2005;353(16):1673-1684. 
63. Piccart-Gebhart MJ, Procter M, Leyland-Jones B, et al. Trastuzumab after adjuvant chemotherapy in HER2-positive breast cancer. $N$ Engl J Med. 2005;353(16):1659-1672.

64. Slamon D, Eiermann W, Robert N, et al. Adjuvant trastuzumab in HER2-positive breast cancer. NEngl J Med. 2011;365(14):1273-1283.

65. Joensuu H, Kellokumpu-Lehtinen PL, Bono P, et al. Adjuvant docetaxel or vinorelbine with or without trastuzumab for breast cancer. $N$ Engl J Med. 2006;354(8):809-820.

66. Spielmann M, Roche H, Delozier T, et al. Trastuzumab for patients with axillary-node-positive breast cancer: results of the FNCLCC-PACS 04 trial. J Clin Oncol. 2009;27(36):6129-6134.

67. Smith I, Procter M, Gelber RD, et al. 2-year follow-up of trastuzumab after adjuvant chemotherapy in HER2-positive breast cancer: a randomised controlled trial. Lancet. 2007;369(9555):29-36.

68. Perez EA, Suman VJ, Davidson NE, et al. Sequential versus concurrent trastuzumab in adjuvant chemotherapy for breast cancer. J Clin Oncol. 2011;29(34):4491-4497.

69. Tolaney SM, Barry WT, Dang CT, et al. Adjuvant paclitaxel and trastuzumab for node-negative, HER2-positive breast cancer. $N$ Engl J Med. 2015;372(2):134-141.

70. Tan-Chiu E, Yothers G, Romond E, et al. Assessment of cardiac dysfunction in a randomized trial comparing doxorubicin and cyclophosphamide followed by paclitaxel, with or without trastuzumab as adjuvant therapy in node-positive, human epidermal growth factor receptor 2-overexpressing breast cancer: NSABP B-31. J Clin Oncol. 2005; 23(31):7811-7819.
71. Perez EA, Suman VJ, Davidson NE, et al. Cardiac safety analysis of doxorubicin and cyclophosphamide followed by paclitaxel with or without trastuzumab in the North Central Cancer Treatment Group N9831 adjuvant breast cancer trial. J Clin Oncol. 2008;26(8): 1231-1238.

72. Russell SD, Blackwell KL, Lawrence J, et al. Independent adjudication of symptomatic heart failure with the use of doxorubicin and cyclophosphamide followed by trastuzumab adjuvant therapy: a combined review of cardiac data from the National Surgical Adjuvant breast and Bowel Project B-31 and the North Central Cancer Treatment Group N9831 clinical trials. J Clin Oncol. 2010;28(21): 3416-3421.

73. Suter TM, Procter M, van Veldhuisen DJ, et al. Trastuzumab-associated cardiac adverse effects in the herceptin adjuvant trial. J Clin Oncol. 2007;25(25):3859-3865.

74. Goldhirsch A, Gelber RD, Piccart-Gebhart MJ, et al. 2 years versus 1 year of adjuvant trastuzumab for HER2-positive breast cancer (HERA): an open-label, randomised controlled trial. Lancet. 2013;382(9897):1021-1028.

75. Pivot X, Suter T, Nabholtz JM, et al. Cardiac toxicity events in the PHARE trial, an adjuvant trastuzumab randomised phase III study. Eur J Cancer. 2015;51(13):1660-1666.

76. Sawaki M, Tokudome N, Mizuno T, et al. Evaluation of trastuzumab without chemotherapy as a post-operative adjuvant therapy in HER2positive elderly breast cancer patients: randomized controlled trial [RESPECT (N-SAS BC07)]. Jpn J Clin Oncol. 2011;41(5):709-712.
Breast Cancer - Targets and Therapy

\section{Publish your work in this journal}

Breast Cancer - Targets and Therapy is an international, peerreviewed open access journal focusing on breast cancer research, identification of therapeutic targets and the optimal use of preventative and integrated treatment interventions to achieve improved outcomes, enhanced survival and quality of life for the cancer patient

\section{Dovepress}

The manuscript management system is completely online and includes a very quick and fair peer-review system, which is all easy to use. Visit http://www.dovepress.com/testimonials.php to read real quotes from published authors. 